\title{
Diálogos e reflexões sobre o conceito de ser vivo: Aplicação do Círculo
}

\section{Hermenêutico-Dialético}

\author{
Dialogues and reflections on the concept of living beings: Application of the Dialetic Hermeneutic
} Circle

\author{
Diálogos y reflexiones sobre el concepto de estar vivo: Aplicación del Círculo Hermenéutico \\ Dialético
}

\begin{abstract}
Resumo
Neste trabalho objetivamos entender as concepções dos discentes de graduação dos cursos de Ciências Biológicas, Educação Física, Enfermagem, Nutrição e Saúde Coletiva acerca do conceito "Ser vivo". Para isso, foram realizadas entrevistas com tais estudantes, utilizando a metodologia do Círculo Hermenêutico-Dialético (CHD). Também aplicamos a nuvem de palavras para enfatizar as possíveis mudanças conceituais. Observou-se que durante a primeira etapa, a maioria dos entrevistados se referiram à este conceito como "ser que tem vida", conceituação que se aproxima da definição disponível no dicionário Aurélio. Entretanto, devido à falta de um conceito unificado, fez-se necessário a aplicação de uma segunda etapa, a qual os entrevistados tiveram acesso às respostas dos demais participantes, para fins de melhoramento ou ressignificação de seus conceitos individuais. Na segunda etapa, três participantes não modificaram suas respostas após o contato com conceitos dos outros entrevistados, já os demais fizeram algumas mudanças no seu conceito sobre ser vivo, no entanto, as respostas dos estudantes ainda continham divergências. Na terceira etapa, os estudantes foram reunidos para propor um consenso final sobre o mesmo assunto, assim houve uma reconstrução positiva, em que as respostas se mostraram coesas e por coincidência, alinhadas com diversos autores da literatura científica. Contudo, o CHD mostrou-se como uma ferramenta eficiente para ensino-aprendizagem de conceitos, podendo ser usada para solucionar e construir um consenso entre grupos e como exercício de discussão sobre temas diversos.
\end{abstract}

Palavras-chave: Conceitualização; Ser vivo; Círculo hermenêutico-dialético. 


\begin{abstract}
In this manuscript, we aim to understand the conceptions of undergraduate students from Biological Sciences, Physical Education, Nursing, Nutrition and Public Health courses about the concept of "Living Being". For this, interviews were conducted with these students, using the methodology of the Hermeneutic-Dialectical Circle (HDC). We also apply the Cloud Words to emphasize possible conceptual changes. It was observed that during the first stage, the majority of interviewees referred to this concept as "being that has life" concept that approaches the definition available in the dictionaries. However, due to the lack of a unified concept, it was necessary to apply a second stage, in which the interviewees had access to the answers of the other participants, in order to improve or re-signify their individual concepts. In the second stage, three participants did not change their answers after being in contact with the other interviewees' concepts, while the others made some changes in their concept of a living being, however, the students' answers still had divergences. In the third stage, students were brought together to propose a final consensus on the same issue, thus there was a positive reconstruction, in which the responses are showed cohesive and coincidentally aligned with several authors of the scientific literature. However, the CHD has proved to be an efficient tool for teaching-learning concepts, and it can be used to solve and build a consensus between groups and as an exercise in discussion on different topics.
\end{abstract}

Keywords: Conceptualization; Living being; Hermeneutic-dialectical circle.

\title{
Resumen
}

En este trabajo buscamos comprender las concepciones de los estudiantes de pregrado de los cursos de Ciencias Biológicas, Educación Física, Enfermería, Nutrición y Salud Pública sobre el concepto de "ser vivo". Para ello, se realizaron entrevistas a dichos estudiantes, utilizando la metodología del Círculo Hermenéutico-Dialéctico (CHD). También aplicamos la nube de palabras para enfatizar posibles cambios conceptuales. Se observó que durante la primera etapa, la mayoría de los encuestados se refirió a este concepto como "ser que tiene vida", concepto que se acerca a la definición disponible en el diccionario Aurélio. Sin embargo, debido a la falta de un concepto unificado, fue necesario aplicar una segunda etapa, en la que los entrevistados tuvieron acceso a las respuestas de los demás participantes, con el fin de mejorar o resignificar sus conceptos individuales. En la segunda etapa, tres participantes no cambiaron sus respuestas luego de contactar con los conceptos de los otros entrevistados, mientras que los demás realizaron algunos cambios en su concepto de ser vivo, sin embargo, las respuestas de los estudiantes aún tenían divergencias. En la tercera etapa, los estudiantes se reunieron para proponer un consenso final sobre el mismo tema, por lo que hubo una reconstrucción positiva, en la que las respuestas se mostraron cohesionadas y por coincidencia, alineadas con varios autores de la literatura científica. Sin embargo, el CHD demostró ser una herramienta eficaz para la enseñanza-aprendizaje de conceptos, pudiendo ser utilizado para resolver y construir consensos entre grupos y como ejercicio de discusión sobre diferentes temas.

Palabras clave: Conceptualización; Ser viviente; Círculo hermenéutico-dialéctico.

\section{Introdução}

O enfoque por técnicas metodológicas que delineiam a percepção e o perfil dos discentes a respeito de temáticas que podem propiciar reflexão e discussão no ensino das Ciências, manifesta desde muito tempo uma atenção significativa no processo de ensino-aprendizagem de conceitos (Villani \& Pacca, 1997). Algumas técnicas e modelos metodológicos podem apresentar embargos no que diz respeito à dimensão coletiva, na perspectiva da heterogeneidade de ideias dentro de um grupo, pois eventualmente se distancia da visão sobre o encontro nas diferenças (Chaluh, 2010). Considerando essa problemática, fica aberta a procura de ferramentas que possibilitam ao processo didático, trabalhar questões importantes de caráter conceitual, através das dimensões dialógica e interativa, inclusive sobre temas da Biologia, cujos diversos objetos de conhecimento denotam alta representação social e sinalizam caminhos na elaboração de práticas educativas, como Saúde e Meio Ambiente (Vittorazzi et al., 2019).

Tendo em vista esta grande abrangência que os conceitos de Biologia podem oferecer para os sujeitos, entende-se a importância da reconstrução conceitual, como coloca David Ausubel (1980), partindo da valorização das concepções prévias no processo de aprendizagem (Ausubel; Novak \& Hanesia, 1980). O conhecimento já apropriado pelo indivíduo pode ter um novo significado quando adjunto à uma metodologia que possibilita a reconstrução de conceitos por meio, por exemplo, do Círculo Hermenêutico-Dialético (CHD), metodologia qualitativa para coleta de dados, proposta por Guba e Lincoln (1989), em que são usados elementos que investigam um consenso confiante da realidade estudada (Neves, 2006; Neves; Carneiro-leão \& Ferreira, 2012). 
O CHD proporciona a união do útil ao agradável no que diz respeito às situações de aprendizagem, promovendo um potente dinamismo entre os indivíduos envolvidos. Trata-se de uma metodologia interativa que envolve o depoimento de estudantes e professores, sendo viável para análise e interpretação de conceitos em diferentes conjecturas, mas com uma visão ampla do tema (Oliveira, 2005). Nesse sentido, através da relação entre a hermenêutica (interpretação) e o dialético (dialogo), é possível o entendimento do textual, da declaração e depoimento dos indivíduos, visto que o CHD contém uma característica de interação e age como mecanismo de mediação do diálogo, reflexão e das discussões entre os envolvidos, além de possibilitar aos sujeitos a análise de suas citações anteriores (Barbosa, 2001; Neves, 2006; Neves; Carneiro-leão \& Ferreira, 2012).

Transcendendo a apuração de dados, o CHD se constitui como um conjunto de meios com referencial pluralistaconstrutivista, ao determinar de forma grupal, diálogos e discussões. É possível a reflexão das variadas apresentações dos achados e formação dos melhores consensos, possibilitando uma boa contribuição para construção e atualização de um conceito (Neves, Carneiro-Leão \& Ferreira, 2012). Através do CHD, o pesquisador pode realizar entrevistas individuais com cada sujeito da pesquisa, possibilitando que suas falas (conceitos) possam ser lidas pelos outros entrevistados, que elaboram uma síntese da sua e das demais respostas. Ao final, esses conceitos podem ou não ser modificados (Oliveira, 2005). Neste trabalho analisamos a concepção de alunos de diferentes cursos de Saúde e Ciências Biológicas, utilizando os recursos do CHD como forma de reflexão, interpretação e discussão sobre a conceituação de ser vivo.

\section{Referencial Teórico}

\subsection{Conceituando ser vivo}

A definição de ser vivo, segundo o dicionário Aurélio, é lidado como aquilo que vive, que tem vida e atividade (Ferreira, 2010). Esta definição assim como o estudo da vida que permeia todo o processo de ensino e aprendizagem da disciplina Biologia, tem uma atenção inicial nos primeiros momentos entre professor e aluno quando se discute as características básicas de um organismo vivo, sendo todo aquele que tem uma organização celular, capacidade de reagir à estímulos, apresenta metabolismo próprio, cresce e gera descendentes através da reprodução. Contudo, ainda que este conceito pareça óbvio, a luz de diferentes perspectivas, pode haver diversos significados e representações. Para psicólogos, trabalha-se a lógica da vida social, para biólogos e outros profissionais, o termo faz referência a florestas, animais e outros estilos de vida (Damineli \& Damineli, 2007).

Segundo Castro (2010), alguns conceitos biológicos são considerados centrais, uma vez que atuam como núcleo por meio do qual outros conceitos podem estar relacionados e serem formados (derivados). O conceito de ser vivo, por exemplo, é considerado um conceito central, já que através dele, conceitos como célula e respiração celular são gerados.

Tomando as definições de central e derivado como eixo importante para entender os diversos significados, o conceito pode ser lidado como uma organização do conhecimento que propõe a identificação, compreensão e seleção de recursos que possam representar o conteúdo, uma vez que relaciona sentidos implícitos de determinado objeto de conhecimento. Dessa maneira, a análise conceitual é lidada como um processo macro, de onde os conceitos selecionados são sintetizados em termos significativos (assuntos), os quais refletem a essência conceitual do objeto informacional (Bräscher \& Melo, 2016).

Por isso que a aprendizagem de um conceito não é uma apropriação instantânea que se faz de algo, mas um processo de construção que ocorre mediado pela linguagem (Carlino, 2013). Para Vygotsky (1998, p. 66-67), "um conceito não é uma formação isolada, fossilizada e imutável, mas sim, uma parte ativa do processo intelectual, constantemente a serviço da comunicação, do entendimento e da solução de problemas". O autor ainda ressalta que o desenvolvimento dos conceitos não espontâneos ou científicos não difere do desenvolvimento dos conceitos cotidianos ou espontâneos dos estudantes, porque os dois processos se relacionam, se influenciam e se movimentam, uniformemente, participando de um único procedimento, o da construção conceitual (VYGOTSKY, 1998). 


\subsection{Círculo Hermenêutico-Dialético}

Conhecida como uma técnica dinâmica, o Círculo Hermenêutico-Dialético (CHD) é utilizada como processo de coleta de dados, dentro de uma abordagem qualitativa, que media um caráter interativo e dinâmico entre o pesquisador e o entrevistado. Esta pesquisa é conhecida por aproximar os envolvidos, porém sem interferência da subjetividade do pesquisador (Oliveira, 2001). Esta técnica metodológica foi criada e usada por Guba e Lincoln (1989), com raízes construtivista e participativa, estabelecendo discussões e diálogos entre grupos, propiciando a análise e reflexão sobre distintos aspectos, tornando possível a troca de conhecimento e a possibilidade de refinamento conceitual (Oliveira, 2005). É uma ferramenta denominada de integrativa (Figura 1), pois parte do princípio da dinâmica do vai-e-vem, já que os conceitos podem ser interpretados, complementados, observados e reinterpretados pelo indivíduo, a partir de premissas e fatos decorridos por outro indivíduo.

Figura 1. Ilustração do processo interativo da metodologia do círculo hermenêutico-dialético, A - aluno entrevistado, C construção teórica, e P - pesquisador. As setas de cor preta indicam o sentido do fluxo da metodologia e as setas de outras cores indicam a troca de informações entre os pesquisados.

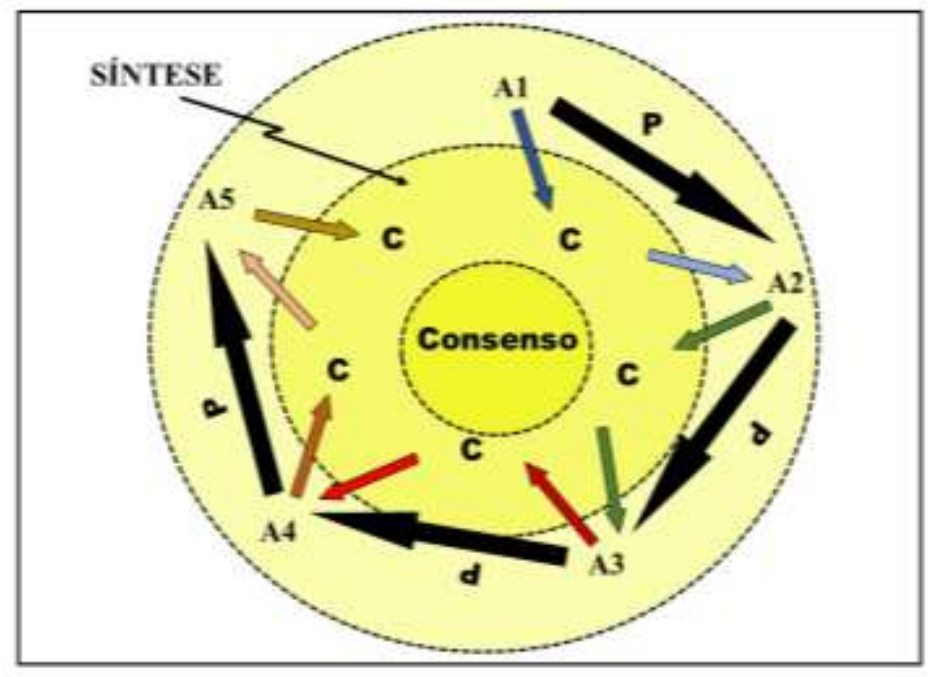

Fonte: Adaptado de Oliveira (1999).

O domínio dialético desta técnica se configura mediante os constantes diálogos, críticas, análises, construções e reconfigurações dos conceitos elaborados pelos participantes durante a coleta de dados. Através desse dinamismo, é possível propiciar um entendimento macro e refinado sobre outras visões da realidade (Oliveira, 2011).

Tal construção e reconstrução, visa a aproximação da realidade pensada, através de entrevistas, questionários, propondo sobretudo, a interação comunicativa por meio do trabalho coletivo entre os participantes, uma vez que é prevalecida a análise da realidade e não um superficial ajustamento e/ou conformidade de ideias, gerando assim, a construção de um novo conhecimento. Além de minimizar a subjetividade do pesquisador, ao final de todas as entrevistas, uma reunião é promovida com todos os participantes para a discussão dos dados obtidos e em seguida, a construção coletiva de uma síntese geral (Guga \& Lincoln, 1989).

\section{Metodologia}

\subsection{Público-alvo}

O CHD foi aplicado em um grupo de cinco graduandos da Universidade Federal de Pernambuco (UFPE), Centro Acadêmico de Vitória (CAV), havendo um representante para cada curso. Os estudantes que representaram os cursos do 
montante da área da Saúde foram: Educação Física, Enfermagem, Nutrição e Saúde Coletiva. O quinto participante era de Ciências Biológicas, denominado como curso de área isolada. Os estudantes foram selecionados por interesse em participar, de forma não probabilística, onde estes foram informados previamente acerca da metodologia utilizada.

\subsection{Aplicação do estudo em campo}

Para aplicação da metodologia de CHD, os discentes foram separados e sem contatos prévios do assunto foi questionado "O que é um ser vivo?". No primeiro momento foi solicitado a cada entrevistado que conceituassem ser vivo, obedecendo um tempo máximo de 5 minutos para responder à pergunta, sem haver consulta em nenhuma fonte de dados (livros ou plataformas online), assim como de outra pessoa. Cada resposta foi digitada no computador do entrevistador. Um segundo momento foi aplicado, permanecendo de forma individual, onde os entrevistados tinham acesso às respostas anteriores dos demais entrevistados, havendo assim, a possibilidade de serem influenciados na manutenção ou melhoramento da segunda resposta destes indivíduos. Na terceira etapa, os entrevistados foram reunidos em uma sala de aula nas dependências do campus, para uma discussão em grupo e construção de conceito único e comum de ser vivo, permeando entre o entendimento e capacidade arguitiva de cada entrevistado.

\subsection{Análise dos dados}

No presente estudo, através do CHD, foram obtidos dados qualitativos, os quais os indivíduos discerniram sobre o tema, principalmente na última etapa da metodologia. Dados quantitativos também foram contemplados, a partir da representação de nuvem de palavras, aquelas que apareceram mais vezes estão exibidas no quadro 2. A abordagem qualitativa serviu para dar enforque na interpretação do objeto de interesse, enquanto a quantitativa proporcionou a compreensão da totalidade dos fenômenos investigados (Fonseca, 2002; Polit, Beck \& Hungler, 2004).

\section{Resultados e Discussão}

Uma das vantagens e utilidades do CHD pode ser a ocultação da identidade do candidato, permitindo que o mesmo realize uma atividade sem receber críticas ou interferência dos demais colegas sobre uma possível resposta incompleta, tendo em vista que todos estejam cientes que o processo é coletivo. Analisando as respostas dos candidatos das cinco áreas, percebese a visão de cada indivíduo e o que eles entendem sobre a questão abordada na atividade. A tabela 1 mostra as respostas da primeira etapa de aplicação do método, onde os entrevistados deram sua primeira concepção sobre o conceito em questão.

Tabela 1. Primeira Etapa: respostas dos entrevistados das áreas de Saúde e Ciências Biológicas por ordem de entrevista sobre o conceito de "ser vivo" em aplicação do Círculo Hermenêutico-Dialético. Resposta 1: percepção individual.

\begin{tabular}{ll}
\hline \multicolumn{1}{c}{ Cursos abordados } & \multicolumn{1}{c}{ Resposta 1 } \\
\hline Ciências Biológicas & Um ser vivo é qualquer coisa composta por células e que é capaz de se reproduzir, tem uma \\
& fisiologia complexa ou não, possa evoluir, possa ocupar um nicho ecológico, etc. \\
Educação Física & São todos os seres que realizam processos bioquímicos para obtenção de energia. \\
Enfermagem & Ser vivo é tudo que consegue respirar. \\
Nutrição & Todos os seres que têm vida, que sentem algo (porque até as bactérias sentem alguma coisa, \\
& nem que seja fome). \\
Saúde Coletiva & Um ser vivo pode ser considerado todos os seres que têm vida, sejam bichos ou plantas. \\
\hline
\end{tabular}


Em concordância com Gelman e Spelka (1983) e Freitas (1989), o desenvolvimento mecânico do conceito de ser vivo é decorrente de uma busca de significado para a expressão "ser vivo", podendo estar diretamente relacionada ao movimento voluntário, respiração, ao ato de se alimentar e até mesmo a capacidade de emitir sons (Varela \& Sá, 2012).

Para Ausubel, Novak e Hanesian (1980), Vygotsky (1998) e Pozo e Crespo (2006), os alunos tendem a definir ser vivo mediante premissas de conceitualização clássica, a qual diz que "todo ser vivo nasce, cresce, reproduz e morre". Nota-se a partir dos dados obtidos na tabela 1, quase todos os entrevistados se referiram a ser vivo como um "ser que tem vida", o que vem de encontro com a própria definição de ser vivo segundo o dicionário Aurélio, com exceção do candidato de Educação Física. Observando separadamente cada percepção de o que vem a ser um ser vivo, podem-se identificar em: O estudante de Ciências Biológicas mencionando "células; reproduzir, fisiologia; nicho ecológico" cada palavra chave citada faz parte de uma disciplina contida no perfil de ensino de Ciências Biológicas do centro acadêmico ao qual ele faz parte. No estudante representante de Nutrição "...nem que seja fome" pode-se interpretar o dizer pelo fato de organismos vivos eventualmente apresentarem necessidade nutricionais. Quanto ao estudante de Educação Física ao que ele se refere como "processos bioquímicos para obtenção de energia" explicita o conhecimento prévio na área de bioquímica que é fortemente trabalhada em sua graduação.

Assim, fez-se necessário a aplicação de uma segunda etapa (Tabela 2), a qual os entrevistados tiveram acesso às respostas dos outros participantes para fins de melhoramento, mudança ou reestruturação do conceito individual escrito na etapa 1, passo este disponível na metodologia do CHD que tem como finalidade incentivar o indivíduo a ter contato com outros olhares acerca do objeto e tomar conclusões distintas, reestruturadas e ou permanecer em suas definições próprias.

Tabela 2. Segunda Etapa: percepção individual com influência da resposta do(os) entrevistado(os) anterior(es) sobre o tema "ser vivo" em aplicação do Círculo Hermenêutico-Dialético. Resposta 2: percepção individual pós permuta de pensamentos. Sem mudança: entrevistados permaneceram com seu conceito inicial sem fazer nenhuma alteração.

\begin{tabular}{ll}
\hline \multicolumn{1}{c}{ Cursos abordados } & Resposta 2 \\
\hline Ciências Biológicas & Sem mudança \\
Educação Física & São todos os seres que realizam processos bioquímicos para \\
& obtenção de energia. Eles nascem, crescem, desenvolvem-se, \\
& reproduzem, envelhecem e morrem. \\
Enfermagem & Sem mudança \\
Nutrição & Sem mudança \\
Saúde Coletiva & Seres que têm vida, se alimentam, respiram e possuem um ciclo \\
& biológico. \\
\hline
\end{tabular}

Fonte: Autores (2021).

A compreensão e desenvolvimento de conceitos partem de premissas empíricas e intrínsecas ao sujeito, haja vista as experiências do cotidiano, contextos sociais e econômicos, bem como a observação da própria natureza (Carrascosa, 2005; Pozo \& Crespo, 2006). Observa-se que três dos participantes não modificaram suas respostas após o contato com conceitos anteriores, e isso já foi apontado por Neves (2006), esclarecendo que isso pode acontecer, e que as modificações teóricas não acontecem explicitamente, mas os conceitos apresentados aos participantes, de alguma maneiras são percebidas e revisadas, contribuindo para um dado resultado. Esses dados informam que ainda há grande divergências do conceito de ser vivo entra as diversas áreas de estudos. Com isso, a metodologia CHD dá a possibilidade de unificação de pensamento com contribuição mútua, neste viés foi tomada como ponto principal as respostas evidenciadas por todos os entrevistados na primeira e segunda 
etapa, solicitando aos mesmos participantes, dialogicamente, unificassem o conceito (Quadro 1), possibilitando e dando ferramentas para reestruturação conceitual com base nas premissas empíricas e conhecimento compartilhado de cada um.

Quadro 1. Terceira Etapa: fichamento conclusivo com influência da resposta de todos entrevistados sobre o tema "ser vivo" em aplicação do Círculo Hermenêutico-Dialético.

Consenso (Resposta única): $\quad$ Ser vivo é todo organismo que possui célula e capaz de realizar um ciclo biológico.

Fonte: Autores (2021).

O debate com todos juntos proporcionaram aos entrevistados novos pontos de vista e perspectivas de pensamento sobre o tema, podendo-se afirmar a partir dos resultados que o aprendizado quando construído em grupo se torna mais enriquecedor do que aprender de forma individual. O consenso final sobre o conceito de ser vivo se mostrou positivo por mostrar uma boa organização das ideias dos participantes e uma interatividade entre os mesmos. Percebeu-se que houve uma reconstrução positiva, em que as respostas se mostraram coesas e próximas daqueles presentes no dicionário e principalmente, alinhadas à vários autores da literatura científica.

É notável a amplitude do conceito sobre ser vivo empiricamente debruçado pelos entrevistados pela abordagem de inúmeras características que até então não são errôneas, se partirmos do ponto que estamos falando de organismos específicos, pois nem todo ser vivo possui todas as características. O Quadro 2 mostra que o conhecimento empírico dos entrevistados é vasto e associa o ser vivo com diversas características, sendo estas amplas e que podem abordar estilos de vidas diferentes e específicas. Quando comparada com o dado conclusivo, podemos observar a abordagem totalmente ampla que envolve características principais que todo ser vivo deve, obrigatoriamente, ter em seu estilo de vida.

Quadro 2. Comparação entre os as palavras-chave observadas nas respostas iniciais e conceito final, através da nuvem de palavras.

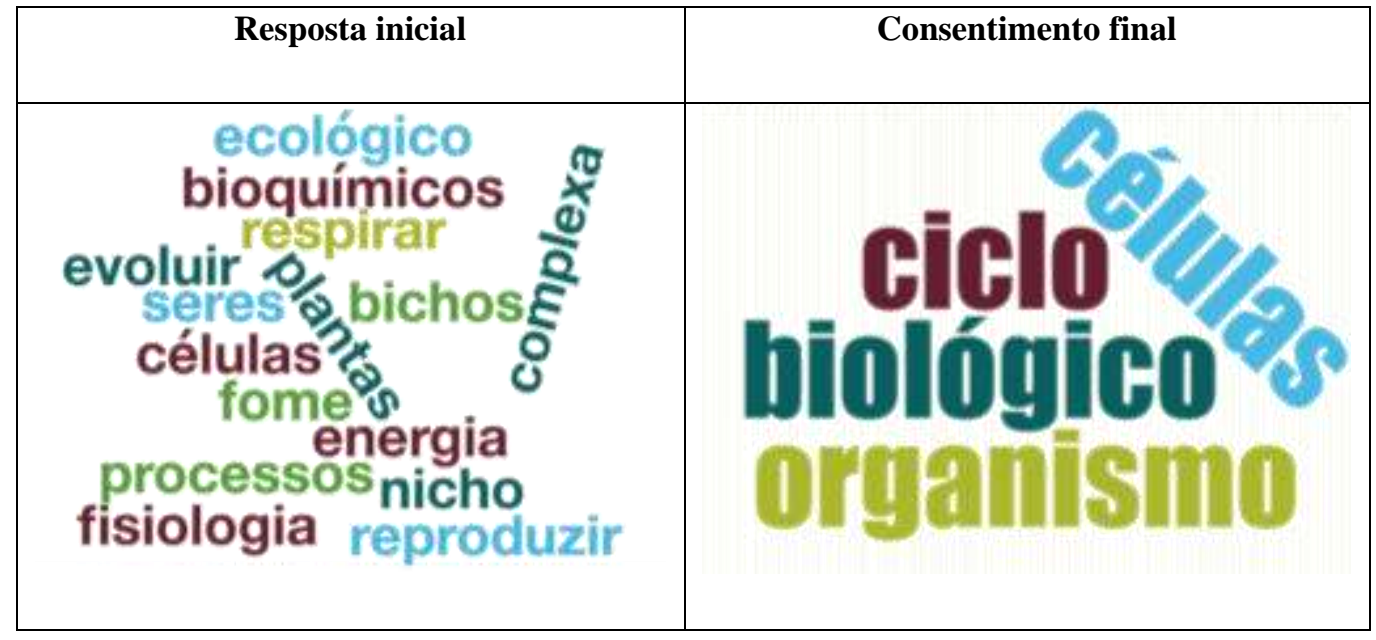

Fonte: Autores (2021).

O conceito de ser vivo é muito complexo, requer atenção e análise em diversas características apresentadas pelo organismo. Segundo Purves, Orians e Heller, (1995) um ser vivo deve permear por diversas características para ser pautado nesta categoria, desde ser organizado por células a possuir um metabolismo que faça uso de energia e realize processos para manutenção de seu platô. Também devem reproduzir, crescer, responder a estímulos, e principalmente evoluir. Sendo assim, a interação, observação e reconstrução dos conceitos feito pelos indivíduos é também sustentada por Araújo et al. (2010), em que considera o CHD um método formidável, ao proporcionar a revisão, construção e reconstrução das falas e com isso possibilita 
uma reflexão sobre o conceito trabalhado.

\section{Considerações Finais}

Através da técnica de entrevistas do CHD, permitiu-se a interação direta entre os entrevistados e os pesquisadores. Os entrevistados estiveram atentos durante as explicações do estudo e nas colocações dos outros participantes, anotaram pontos importantes para eles, reconstruíram suas concepções, modificaram suas visões e conceitos, e apresentaram uma ótima desenvoltura nas discussões, mostrando novos argumentos para questionamentos e reflexões a partir do CHD. Como não é possível avaliar a forma e intensidade que o conteúdo foi trabalhado em outros momentos da vida escolar/acadêmica destes indivíduos, pode-se levar em consideração que cada um deles tem uma visão distinta de mundo a partir de suas experiências pessoais e nível de relação com suas áreas de conhecimento específicas.

\section{Referências}

Araújo, A. F., Nogueira, R. A., Oliveira; M. M., \& Silva, J. P. G. (2010). Círculo Hermenêutico-Dialético como ferramenta didática para trabalhar as questões relativas à educação ambiental. $62^{\circ}$ Reunião Anual da SBPC, Natal, Rio Grande do Norte, Brasil.

Ausubel, D. P., Novak, J. D. \& Hanesian, H. (1980). Psicologia educacional. (2a ed.), Interamericana.

Barbosa, R. H. S. (2001). Mulheres, reprodução e AIDS: as tramas da ideologia na assistência à saúde de gestantes HIV+. Tese de doutorado - Escola Nacional de Saúde Pública, Rio de Janeiro, Brasil.

Brasil. (2000). Parâmetros Curriculares Nacionais: Ciências da Natureza, Matemática e suas Tecnologias/Secretaria de Educação Fundamental. Brasília: $\mathrm{MEC} / \mathrm{SEF}$.

Carlino, E. P. (2013). Pensando sobre a aprendizagem de conceitos em sala de aula. Santa Maria, 38(1), 137-150.

Carrascosa, J. (2005). El problema de las concepciones alternativas en la actualidad (parte I). Análisis sobre las causas que las originan y/o mantienen. Revista Eureka sobre Enseñanza y Divulgación de las Ciencias, 2(2), 183-208.

Castro, D. R. (2010). Estudo de Conceitos de Seres Vivos nas Séries Iniciais. Dissertação de Mestrado - Universidade Federal da Bahia. Salvador, Brasil.

Chaluh, L. N. (2010). Do trabalho coletivo na escola: encontros na diferença. Pro-Posições, 21(2), 207-223.

Damineli, A. \& Damineli, D. S. C. (2007). Origens da vida. Estudos avançados, 21 (59).

Ferreira, A. B. H. (1995). Novo dicionário Aurélio da língua portuguesa. (3a ed.), Obra em 19 fascículos semanais encartados na Folha de São Paulo, de outubro de 1994 a fevereiro de 1995.

Ferreira, A. B. H. (2010). Dicionário da língua portuguesa. (5a ed.), Positivo. 2222 p.

Fonseca, J. J. S. (2002). Metodologia da pesquisa científica. UEC, Apostila.

Freitas, M. (1993). Distinção entre ser vivo e ser inanimado: uma evolução por estádios ou um problema de concepções alternativas? Revista Portuguesa de Educação, 2(1), 33-50.

Gelman, R. \& Spelke, E. (1993). The development of thoughts about animate and inanimate objects: implications for research on social cognition. In J. Flavell \& L. Ross (Ed.), Social Cognitive Development. Cambridge University Press, Cambridge, Reino Unido.

Guba, E. \& Lincoln, Y. S. (1989). Fourth generation evaluation. Sage.

Melo, M. A. F. \& Bräscher, M. (2014). Termo, conceito e relações conceituais: um estudo das propostas de Dahlberg e Hjorland. Ci. Inf., Brasília, 41(1), 6780 .

Minayo, M. C. S. (org.). (2001). Pesquisa Social. Teoria, método e criatividade. (18a ed.), Vozes.

Moreira, M. A. (1999). Teorias de Aprendizagem. EPU.

Neves, R. F., Carneiro-Leão, A. M. A. \& Ferreira, H. S. (2012). A interação do Ciclo da Experiência de Kelly com o Círculo Hermenêutico-Dialético para a Construção de Conceitos de Biologia. Ciência \& Educação, 18(2), 335-352.

Oliveira, M. M. (2011). Círculo hermenêutico-dialético como sequência didática interativa. Revista Interfaces Brasil/Canadá, 11(1), $235-251$.

Oliveira, M. M. (1999). Formação em associativismo e desenvolvimento no Nordeste do Brasil: a experiência de Camaragibe. Tese de Doutorado em Educação- Universidade de Sherbrooke, Canadá.

Oliveira, M. M. (2001). Metodologia Interativa: um processo hermenêutico dialético. Interfaces Brasil/Canadá, 1(1), 67-78. 
Research, Society and Development, v. 10, n. 8, e32510817469, 2021

(CC BY 4.0) | ISSN 2525-3409 | DOI: http://dx.doi.org/10.33448/rsd-v10i8.17469

Oliveira, M. M. (2005). Como fazer Pesquisa Qualitativa. Ed. Bagaço. 192 p.

Oliveira, M. M. (2015). Metodologia interativa: um processo hermenêutico dialético. Revista Brasileira de Estudos Canadenses, 1(1), 67-80.

Polit, D. F., Beck, C. T. \& Hungler, B. P. (2004). Fundamentos de pesquisa em enfermagem: métodos, avaliação e utilização. Trad. de Ana Thorell. (5a ed.), Porto Alegre: Artmed.

Pozo, J. I. \& Crespo, M. A. (2006). Aprender y Enseñar Ciencia. Madrid: Ediciones Morata, S.L.

Purves, W. K., Orians, G. H. \& Heller, H. C (1995). "Characteristics of Living Organisms." In Life: The Science of Biology, 1-3. (4a ed.), Sunderland, MA: Sinauer Associates.

Varela, P. \& Sa, J. (2012). Ensino experimental reflexivo das ciências: uma visão crítica da perspectiva piagetiana sobre o desenvolvimento do conceito de ser vivo. Investigações em Ensino de Ciências, 17(3), 547-569.

Villani, A., \& Pacca, J. L. D. A. (1997). Construtivismo, conhecimento científico e habilidade didática no ensino de ciências. Revista da faculdade de Educação, 23(1-2).

Vittorazzi, D. L., Freitas, T. S., Gouveia, D. S. M., Fejolo, T. B. \& Silva, A. M. T. B. (2019). Os temas de Biologia sob a ótica das representações sociais nas pesquisas em Ensino de Ciências: um levantamento no cenário nacional. Ensino, Saúde e Ambiente, 12(1), 1-20.

Vygotsky, L. S. (1998). Pensamento e linguagem. (2a ed.), Martins Fontes Editora. 